\title{
Síndrome hemofagocítico secundario a leishmaniasis visceral en un lactante mayor: reporte de caso
}

\section{Hemophagocytic sindrome secondary to visceral leishmaniasis in a toddler: report of a case}

\author{
Vanessa G. Alvarado-Claros ${ }^{1, a}$, Victoria G. Nieto-Zelaya ${ }^{1, a}$, Sandra N. Tovar- \\ Calderon 2,b,c, Juan José Navarro2,b \\ 1 Universidad Nacional Autónoma de Honduras. Tegucigalpa, Honduras. \\ 2 Sala de Nutrición, Hospital Escuela Universitario. Tegucigalpa, Honduras. \\ a Doctora en Medicina y Cirugía, b Médico Pediatra, c Gastroenteróloga pediatra
}

\section{Correspondencia}

Vanessa Gabriela Alvarado Claros vanclaros24@gmail.com

Recibido: $12 / 05 / 2017$

Arbitrado por pares

Aprobado: 02/08/2017

Citar como: Alvarado-Claros VG, Nieto-Zelaya VG, TovarCalderon SN, Navarro JJ. Síndrome hemofagocítico secundario a leishmaniasis visceral en un lactante mayor: reporte de caso. Acta Med Peru. 2017;34(4):328-32

\section{RESUMEN}

El síndrome hemofagocítico está caracterizado por la activación anómala del sistema inmune. De etiología primaria o secundaria a enfermedades infecciosas, metabólicas, inmunitarias o neoplásicas. Con incidencia de 0,12 a 1 casos por cada 100000 niños y una sobrevida de dos meses de no recibir tratamiento. Se diagnostica con 5 de 8 criterios propuestos por la Asociación Internacional de Histocitosis, y tratamiento según etiología. . Se presenta el caso de un paciente masculino de 15 meses de edad, procedente una zona endémica de leishmaniasis (Choluteca, Honduras), que ingresó al Hospital Escuela Universitario con cuadro clínico de fiebre prolongada y hepatoesplenomegalia. En los exámenes auxiliares de detectó: pancitopenia, serología positiva para leishmania, hipertrigliceridemia y valor de ferritina aumentada. Se hizo el diagnóstico de leishmaniasis visceral y síndrome hemofagocítico. Se trató con antimoniato de meglumina por 28 días, metilprednisolona y prednisona; con evolución favorable. El síndrome hemofagocítico podría ocurrir secundario a leishmaniasis visceral por lo que su diagnóstico debería sospecharse tempranamente para un tratamiento oportuno y mejor pronóstico.

Palabras clave: Linfohistiocitosis hemofagocítica; Leishmaniasis visceral; Síndrome hemofagocítico reactivo; Antimonio (fuente: DeCS BIREME).

\section{ABSTRACT}

The hemophagocytic syndrome is characterized by an abnormal activation of the immune system. This condition may be primary or secondary to infectious, metabolic, immunological, or malignant conditions. Its incidence is 0.12 to 1 case per 100,000 children, and the survival time is around two months with no therapy. The condition is diagnosed with the occurrence of 5 of 8 criteria proposed by the International Histiocytosis Association, and its therapy is according the original etiology. We present the case of a 15-month old male patient, who was brought from Choluteca (an endemic area for leishmaniasis), who was admitted to the University Hospital with prolonged fever and hepatosplenomegaly. Laboratory tests showed pancytopenia, positive serology for Leishmania, hypertriglyceridemia, and increased ferritin levels. Visceral leishmaniasis and hemophagocytic syndrome were diagnosed. The patient received meglumine antimoniate for 28 days, and also methylprednisolone and prednisone; and he had a favorable outcome. The hemophagocytic syndrome may be secondary to visceral leishmaniasis, and its diagnosis should be promptly suspected in order to have timely therapy and a better prognosis. Keywords: Lymphohistiocytosis, hemophagocytic; Leishmaniasis, visceral; Hemophagocytic syndrome, reactive; Antimony (source: MeSH NLM). 


\section{INTRODUCCIÓN}

El síndrome hemofagocítico (SH), también denominado linfohistioctosis hemofagocítica, es un trastorno hemofagocítico caracterizado por la activación y proliferación no maligna del sistema inmune ${ }^{[1-3]}$. Ocurre por la activación anómala de los linfocitos $\mathrm{T}$, células natural killer y productos de citoquinas ${ }^{[4]}, \mathrm{Y}$ puede ser primario (familiar) y secundario. La forma primaria está ligada a factores genéticos autosómicos recesivos y la secundaria se presenta subsiguiente a enfermedades infecciosas, genéticas, neoplásicas, metabólicas o inmunitarias ${ }^{[5]}$.

Reportes multicéntricos informan una incidencia estimada de 0,12 a 1 casos por cada 100000 niños por año, aunque sólo 1 de 3 pacientes se diagnostica antes de su fallecimiento, por lo que la incidencia real sería mayor ${ }^{[4,6]}$, presenta una sobrevida promedio de dos meses en caso no recibir tratamiento ${ }^{[5]}$ y una mortalidad de 46,4\% en Latinoamérica, correspondiendo en su mayoría a casos asociados a infecciones ${ }^{[6]}$. Según la literatura se han reportado solamente dos casos en Honduras ${ }^{[7,8]}$.

Se considera importante la presentación de un caso de leishmaniasis visceral, asociada con un síndrome hemofagocítico; complicación poco usual pero no desestimable ${ }^{[9]}$, en el cual su diagnóstico está sujeto a la alta sospecha, y que realizado en forma temprana favorece al tratamiento oportuno y una evolución satisfactoria.

\section{REPORTE DE CASO}

Paciente masculino de 15 meses de edad, procedente de zona rural del departamento de Choluteca, Honduras (zona endémica de leishmaniasis); producto de un séptimo embarazo. Sin antecedentes de importancia. Madre niega algún grado de consanguinidad con el padre del niño.

El paciente fue ingresado al Hospital Escuela Universitario (Tegucigalpa, Honduras) con historia de fiebre no cuantificada de 15 días de evolución, continua, acompañada de sudoración, escalofríos, aumento progresivo del perímetro abdominal y evacuaciones diarreicas. A los seis días de iniciada la sintomatología, el niño recibió manejo ambulatorio, en un centro de atención primaria de su localidad, con antipirético (acetaminofén) y antibiótico (amoxicilina) durante cinco días sin mejoría clínica, por lo que fue referido al Hospital General del Sur (Choluteca, Honduras) donde permaneció por cuatro días. Los exámenes realizados en este lugar reportaron pancitopenia (leucopenia, trombocitopenia y anemia), siendo referido para diagnóstico y manejo especializado por probable síndrome mieloproliferativo.

A la exploración física se encontró una frecuencia cardíaca de 138 latidos por minuto, frecuencia respiratoria 49 respiraciones por minuto, temperatura $36,5^{\circ} \mathrm{C}$; las variables antropométricas fueron: peso $7,8 \mathrm{~kg}$, talla $73 \mathrm{~cm}$, perímetro cefálico: $44 \mathrm{~cm}$, índice de peso/talla: $-2,-3$ desviaciones estándar (DE) en el puntaje $Z$ y talla/edad: $-2,-3 D E$ en el puntaje $Z$ de acuerdo a las curvas de crecimiento de la Organización Mundial de la Salud ${ }^{[10]}$. Se observó despierto, reactivo a estímulo externo, irritable; palidez generalizada, piel seca y descamada con predominio en cara y extremidades. Abdomen sin evidencia de circulación colateral, con hepatomegalia de $5 \mathrm{~cm}$ por debajo del reborde costal derecho y esplenomegalia de $10 \mathrm{~cm}$ por debajo del reborde costal. Resto de examen físico sin alteraciones. Exámenes de laboratorio: ver Tabla 1.

Considerando los hallazgos clínicos, epidemiológicos y de laboratorio se diagnosticó leishmaniasis visceral asociada a $\mathrm{SH}$, desnutrición crónica agudizada, infección urinaria.

En su cuarto día intrahospitalario, el niño presentó disnea leve, a la auscultación se evidenció presencia de roncus y estertores bilaterales, temperatura $40^{\circ} \mathrm{C}$, además se encontró infiltrado basal derecho en la radiografía de tórax, haciéndose el diagnóstico de neumonía asociada a los centros de atención de salud.

La medicación incluyó el uso de antimoniato de meglumina intramuscular por 28 días, a los 15 días de inicio del medicamento, se indicó metilprednisolona vía intravenosa a $30 \mathrm{mg} / \mathrm{kg} /$ día por tres días y prednisona vía oral a $1 \mathrm{mg} / \mathrm{kg} /$ día por tres días. Además, se le indicó el uso de antibióticos de amplio espectro como: ceftriaxona (cefalosporina de tercera generación) a 75 $\mathrm{mg} / \mathrm{kg} /$ día y oxacilina (betalactámico) a $100 \mathrm{mg} / \mathrm{kg} /$ día, por vía intravenosa durante diez días y se transfundió glóbulos rojos empacados para la anemia microcítica hipocrómica. Presentó mejoría clínica evidente al concluir el tratamiento. Se dio de alta a los 35 días con seguimiento por consulta externa.

\section{DISCUSIÓN}

El síndrome hemofagocítico puede ser secundario a enfermedades infecciosas, entre ellas las más frecuentes son mononucleosis infecciosa y leishmaniasis. De esta última, se ha documentado el SH como una complicación que se presenta en un 13-17\% ${ }^{[11,12]}$. Generalmente pasa desapercibido ${ }^{[4]}$ debido a que agrupa diversas enfermedades con hallazgos clínicos y de laboratorio similares ${ }^{[13]}$. El caso presentado es sobre un lactante mayor que proviene de zona endémica de leishmaniasis, que cumplió con los criterios para sospechar linfohistiocitosis como una complicación.

Clínicamente el SH se presenta con fiebre prolongada (mayor de siete días) ${ }^{[14]}$ que no responde a antibióticos, hepatoesplenomegalia, síntomas neurológicos como irritabilidad, adenopatías, disminución del estado de conciencia y convulsiones. También pueden tener alteraciones dermatológicas como ictericia, rash, exantema maculopapular eritematoso y eritrodermia [5]. La mayoría de las manifestaciones clínicas mencionadas se presentaron en este caso, las cuales también pueden encontrarse en otras enfermedades infecciosas, neoplásicas, inmunes o metabólicas ${ }^{[1-4]}$; también en complicaciones como sepsis, síndrome de respuesta inflamatoria sistémica y síndrome de disfunción multiorgánica ${ }^{[13]}$, evidenciando así la dificultad del diagnóstico. 
Tabla 1. Exámenes de laboratorio. Caso síndrome hemofagocítico secundario a leishmaniasis visceral.

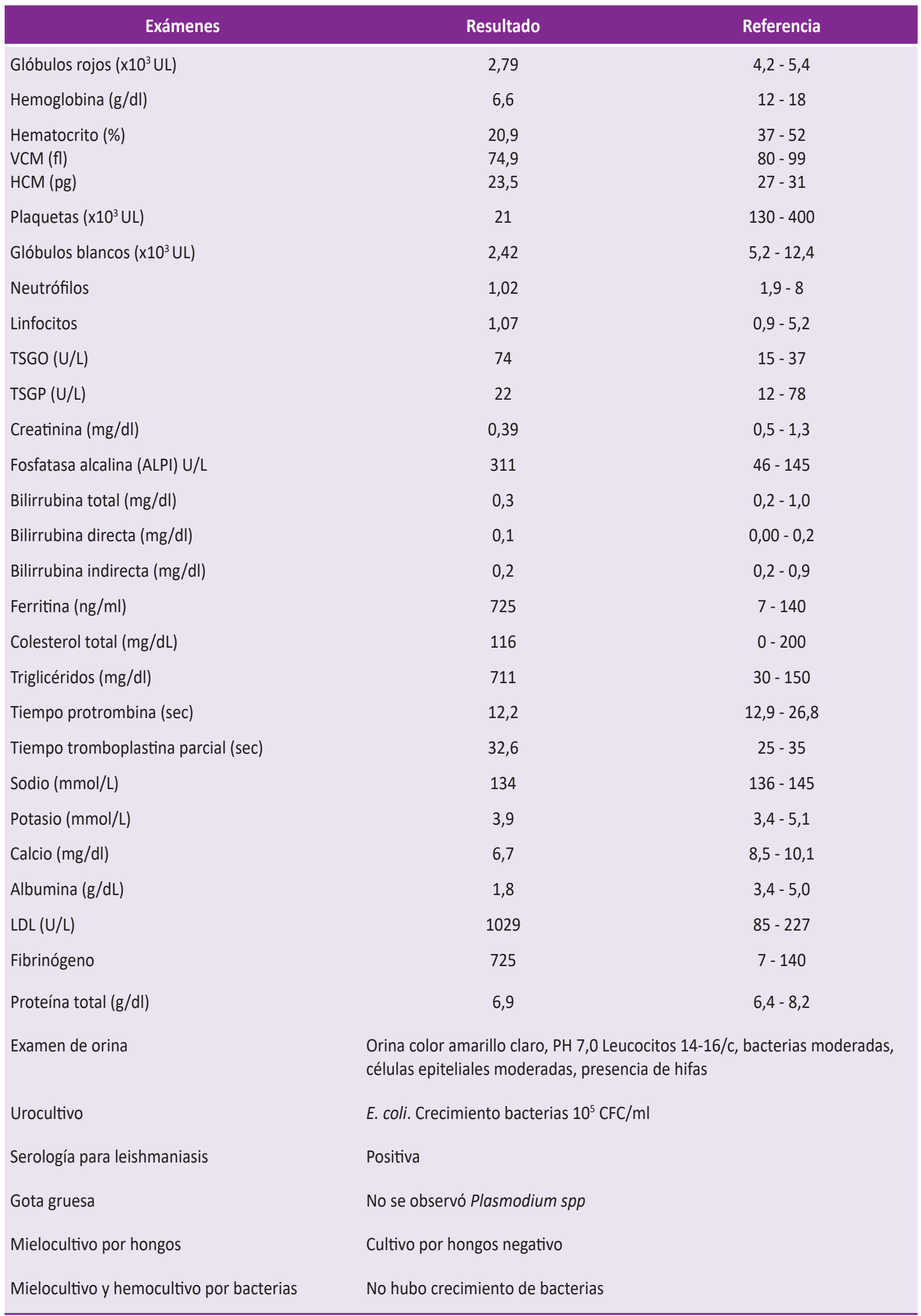


Tabla 2. Criterios diagnósticos para síndrome hemofagocítico. International Histiocyte Society HLH-2004 ${ }^{[5,18]}$.

Criterios diagnósticos

El diagnóstico se establece si uno o dos de los criterios se cumplen:

- Diagnóstico molecular consistente con SH.

- Criterios diagnósticos para SHF (al menos cinco criterios):

1. Fiebre $>$ ó $=38.5^{\circ} \mathrm{C}$

2. Esplenomegalia.

3. Citopenias (afecten 2 o 3 líneas en sangre periférica):

- Hemoglobina < $9 \mathrm{mg} / \mathrm{dL}$ (en niños < 4 semanas: hemoglobina < $100 \mathrm{~g} / \mathrm{L}$ ).

- Plaquetas $<100,000 / \mathrm{L}$

- Neutrófilos $<1,000 \mathrm{~L}$

4. Hipertrigliceridemia y/o hipofibrinogenemia.

- Triglicéridos en ayuno $>265 \mathrm{mg} / \mathrm{dL}$.

- Fibrinógeno $<1.5 \mathrm{~g} / \mathrm{L}$

5. Hemofagocitosis en medula ósea, ganglios, hígado o bazo: no evidencia de malignidad.

6. Niveles bajos o ausencia de actividad de células NK.

7. Ferritina $>500 \mu \mathrm{g} / \mathrm{l}$.

8. $\mathrm{CD} 25$ soluble $>2,400 \mathrm{U} / \mathrm{ml}$.

Comentarios:

Si la hemofagocitosis no se encuentra al momento de presentación, se debe buscar posteriormente. Si la medula ósea no es concluyente, se debe buscar en otros órganos. Múltiples aspirados de medula ósea pueden ser de ayuda para confirmar el diagnóstico.

Los siguientes hallazgos pueden ser evidencia de soporte para el diagnóstico: a) pleocitosis en LCR (células mononucleares) y/o proteinorraquia, y b) biopsia de hígado con hepatitis crónica persistente.

Otros hallazgos clínicos o de laboratorio consistentes con el diagnostico son: síntomas cerebromeningeos, linfadenopatia, ictericia, edema, rash, alteración en las enzimas hepáticas, hipoproteinemia, hiponatremia, VLDL alta, HDL baja.

VLDL: lipoproteínas de muy baja densidad; HDL: lipoproteínas de alta densidad.

El diagnóstico de SH requiere cinco de ocho criterios propuestos por la Sociedad Internacional de Histiocitosis (2004) ${ }^{[5]}$ (ver Tabla 2); sin embargo, inicialmente no todos los criterios pueden estar presentes. La forma primaria se confirma mediante la evidencia de mutaciones en genes como: PFR1, UNC13D, Munc 18-2, Rab27a, STX11, SH2D1A, BIRC4 ${ }^{[5,15]}$, pruebas no disponibles en este país.

Se descartó asociación familiar y episodios recurrentes ${ }^{[4]}$ considerándose un síndrome de etiología secundaria por Leishmania spp, el cual junto al virus de Epstein-Bar son los agentes etiológicos de mayor frecuencia en la linfohistiocitosis ${ }^{[4,11]}$ siendo una complicación común en zonas donde es endémica la leishmaniasis ${ }^{[16-18]}$. El paciente presentó cinco de ocho criterios confirmados por clínica y laboratorio (fiebre prolongada, hepatoesplenomegalia, pancitopenia, hipertriglicéridemia, elevación de ferritina sérica); asimismo se destaca que la ausencia de hemofagocitosis en médula ósea no es suficiente para descartar el diagnóstico y se recomienda la realización de múltiples aspirados de médula ósea cuando se sospecha el $\mathrm{SH}^{[5,19]}$. Ante el diagnostico debe actuarse rápidamente por ser un síndrome con una sobrevida de dos meses de no recibir tratamiento ${ }^{[5]}$.

El tratamiento de las formas secundarias, consiste en el manejo de la infección presente, pero esto puede no ser suficiente y se deberá utilizar el protocolo HLH-2014 que consiste en etopósido
(VP-16) dexamentasona y ciclosporinas y si existe compromiso neurológico debe recibir terapia intratecal con metrotexato y dexametasona ${ }^{[18]}$. En este niño además del tratamiento de la infección subyacente (leishmaniasis) para el que se utilizó antimoniato de meglumina (425 mg de antimonio base por ampolla de $5 \mathrm{ml}$ ) droga disponible en nuestro medio, también requirió el uso de esteroides para una respuesta más eficaz.

En conclusión, el SH puede ser secundario a procesos infecciosos como leishmaniasis visceral, por lo que su sospecha temprana y diagnóstico oportuno, nos permite realizar un tratamiento eficaz, que eviten complicaciones más graves o incluso la muerte.

Fuente de financiamiento: El autor declara no haber recibido ninguna financiación para la realización de este trabajo.

Declaración de conflicto de intereses: Los autores declaran no tener conflicto de intereses con la publicación de este artículo.

\section{REFERENCIAS BIBLIOGRÁFICAS}

1. Young P, Peroni J, Finn BC, Venditti JE, Preiti V, Bullorsky E, et al. Síndrome hemofagocítico. Reporte de 4 casos y revisión de la literatura. Rev Med Chile. 2011;139(2):224-9.

2. Peña C, Valladares $X$, Cabrera M. Síndrome hemofagocítico secundario: reporte de 5 casos. Rev Med Chile. 2013;141(11):1475-9. 
3. Martínez I, Fernández B, Valentinc J, Castillo C, Chamorro E, Pérez-Martínez A. La actividad citotóxica de las células Natural killer como herramienta diagnostica en pacientes pediátricos críticos con sospecha de síndrome hemofagocítico. Med Intensiva. 2015;39(4):213-21.

4. Pérez-Martínez A. Síndrome hemofagocítico (I): concepto, clasificación, fisiopatología y clínica. An Pediatr Contin. 2013;11(5):237-44

5. Santidrian V, Rosso D, Elena G. Síndrome hemofagocítico en pediatría; Revisión Bibliográfica. Revista Pediátrica Elizalde. 2013;4(1):18-22.

6. Estrella D, Pérez M, Alcántar A, Reyes G. Características clínicas del síndrome hemofagocítico en niños sonorenses. Bol Clin Hosp Infant Edo Son. 2016;33(2):49-53.

7. Espinal D, Salinas F, Lanza L. Reposte de caso: linfohistiocitosis hemofagocitica adquirida. Scientifica, 2016;14(1):38-41.

8. Izaguirre-González A, Sánchez-Sierra L, Cerrato-Castro A, Flores-Irias J, Peña A. Síndrome hemofagocítico reactivo en Lactante mayor: Reposte de caso. Arch Med. 2016;12(3):16-21.

9. Apodaca S, Martinez de Cuellar C, Lovera D, Arbo A. Comportamiento clínico de la leishmaniosis visceral en población infantil. Diez años de experiencia de un centro de referencia del Paraguay. Rev Inst Med Trop. 2015;10(1):12-8.

10. Secretaria de Salud de Honduras. Normas para la vigilancia nutricional de los niños y niñas menores de 5 años de edad. Honduras: Secretaria de Salud; 2012.
11. Mahmud I, Marchi A, Lopes M, Malvera R, Irrazabal S, Galarza A. Leishmaniasis visceral infantil. Rev Contexto Saúde. 2013;13(3):27-36.

12. Apodaca S, Araya S, Lovera D, Delgado V, Sanabria G, Arbo A. Leishmaniasis Visceral en niños inmucompetentes. Rev Inst Med Trop. 2014;9(1):28-41.

13. Pérez-Martínez A, Síndrome hemofagocítico (II): diagnóstico y tratamiento. An Pediatr Contin. 2013;11(5):245-53.

14. Porras $\mathrm{O}$. Linfohistiocitosis hemofagocítica, el espectro desde la enfermedad genética al síndrome de activación macrofagocítica. Acta Med Costarric. 2011;53(2):71-8.

15. Espinoza K, Garcia P, Fossas D, León E. Síndrome hemofagocítico. Conceptos actuales. Gac Med Mex. 2013;149(4):431-7.

16. Sotoca J, García L, Lillo M, García O, Carrascosa M, Tébar R. Síndrome hemofagocítico secundario a leishmaniasis visceral. An Pediatr. 2008;69(1):46-8.

17. Instituto de Enfermedades Infecciosas y Parasitología Antonio Vidal. Manual de Manejo de Enfermedades Parasitarias Prioritarias en C.H. Honduras. Instituto de Enfermedades Infecciosas y Parasitología. Antonio Vidal. --2a. Ed. Tegucigalpa: Organización Panamericana de la Salud; 2009.

18. Dapena J, Díaz C, Bastida P, Llort A, Álvarez E, Olive T, Sánchez J. Síndrome hemofagocítico: expresión de diversas entidades nosológicas. An Pediatr. 2009;71(2):110-6.

19. Henter JI, Horne A, Aricó M, Egeler RM, Filipovich AH, Imashuku $\mathrm{S}$, et al. HLH-2004: Diagnostic and therapeutic guidelines for hemophagocytic lymphohistiocytosis. Pediatr Blood Cancer. 2007;48(2):124-31.

\section{Ahora puede enviar sus artículos para Acta Médica Peruana en nuestro Open Journal System:}

\section{www.amp.cmp.org.pe}

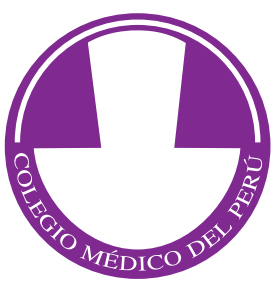

